better to an intravenous regimen. We can only record, anecdotally, that he showed a complete recovery without neurological deficit.

We have not obtained significant blood pressure reduction over two hours in severe hypertension with $80 \mathrm{mg}$ propranolol or $100 \mathrm{mg}$ atenolol by mouth, and bradycardia may be troublesome with these drugs. Further details from Dr Good of the dose, drugs, rate of blood pressure fall, and cardiovascular effects of oral beta-blockade would provide valuable comparison with our data.

R R GHose

$M$ UPADHYHAY

Singleton Hospital,
Swansea

W D MORGAN

\section{Do people smoke for nicotine?}

SIR,-Mr Robert Stepney (8 April, p 922) noted that as the average nicotine delivery of UK cigarettes declined the annual consumption of manufactured cigarettes per smoker increased greatly. This observation suggested that a smoker may compensate for reduced nicotine deliveries by altering the way individual cigarettes are smoked. Your correspondent was careful to point out that a possible alternative explanation is that the number of smokers giving up the habit may have been drawn more from light than from heavy smokers, but submitted that until this is shown to have occurred the association between reduced nicotine delivery and increased consumption will tend to support the nicotinedependence view of smoking (but see also Professor C M Fletcher, 29 April, p 1143).

The purpose of this letter is to corroborate the point made by Mr Stepney, using Canadian data. These also indicate that the annual consumption of manufactured cigarettes per smoker has increased as nicotine deliveries have decreased. While survey data indicate that the percentage of cigarette smokers has decreased, lighter smokers are diminishing as a class. ${ }^{1}$ However, for most age-sex groups the median number of "constant tar cigarettes" smoked has decreased. ${ }^{2}$ These survey data may represent underestimates, but nevertheless since tar and nicotine deliveries are highly correlated they are consistent with the assumption that many smokers can adjust to a cigarette delivering less nicotine.

Relevant evidence is also available from British data, summarised in the table below, which indicates that both male and female smokers have been smoking more cigarettes but that relatively more female ex-smokers are drawn from the light-smoking class. This fact may explain some of the increase in amount smoked per smoker noted by $\mathrm{Mr}$ Stepney.

Distribution of estimated daily amounts smoked in percentages (rounded) by current manufactured cigarette smokers and by current ex-smokers at the time of stopping, Britain 1965, 1971, 19754

\begin{tabular}{|c|c|c|c|c|c|c|c|}
\hline \multirow{2}{*}{\multicolumn{2}{|c|}{ Consumption }} & \multicolumn{2}{|c|}{1965} & \multicolumn{2}{|c|}{1971} & \multicolumn{2}{|c|}{1975} \\
\hline & & Smokers & Ex-smokers & Smokers & Ex-smokers & Smokers & Ex-smokers \\
\hline \multicolumn{8}{|c|}{ Men } \\
\hline $\begin{array}{r}<11 \text { cigarettes } \\
11-25 \text { cigarettes } \\
>25 \text { cigarettes }\end{array}$ & $\begin{array}{l}. \\
\because \\
.\end{array}$ & $\begin{array}{l}32 \\
50 \\
18\end{array}$ & $\begin{array}{l}29 \\
43 \\
28\end{array}$ & $\begin{array}{l}26 \\
50 \\
24\end{array}$ & $\begin{array}{l}27 \\
45 \\
29\end{array}$ & $\begin{array}{l}23 \\
47 \\
30\end{array}$ & $\begin{array}{l}24 \\
45 \\
30\end{array}$ \\
\hline \multicolumn{8}{|c|}{ Women } \\
\hline $\begin{array}{r}<11 \text { cigarettes } \\
11-25 \text { cigarettes } \\
>25 \text { cigarettes }\end{array}$ & $\begin{array}{l}. \\
\because .\end{array}$ & $\begin{array}{l}55 \\
34^{*} \\
10 \dagger\end{array}$ & $\begin{array}{l}67 \\
23^{*} \\
10 \dagger\end{array}$ & $\begin{array}{r}44 \\
45 \\
9\end{array}$ & $\begin{array}{r}67 \\
26 \\
8\end{array}$ & $\begin{array}{l}36 \\
50 \\
13\end{array}$ & $\begin{array}{r}54 \\
36 \\
9\end{array}$ \\
\hline
\end{tabular}

*Percentage for 11-20 cigarettes; tpercentage for $21+$ cigarettes.
Lastly, it may be noted from studies on individual smokers that there are indications that the majority of smokers can adjust to a cigarette delivering less nicotine. ${ }^{3}$ That is, while many smokers changing to a cigarette delivering less nicotine tend to smoke so as to obtain more nicotine, it has been found that their mouth-level exposure, is still generally considerably less than if they were to smoke their regular cigarette. Hence these and related observations suggest that it seems desirable to continue with the various measures, and also to consider additional measures, to make available cigarettes delivering less nicotine and tar.

\section{Department of Statistics, Waterloo, Ontario}

${ }^{1}$ Ouellet, B L, Romeder, J-M, and Lance, J-M, Premature Mortality Attributable to Smoking and Hazardous Drinking in Canada, vol 1, 14. Staff Thompson, M E, Statistics of Smoking in Canada. 1978

Forbes, W F, et al, International fournal of the Addictions, 1976, 4, 933

Lee, P N, Statistics of Smoking in the United Kingdom. 7th edn, tables $50 \mathrm{~W}$ and $23 \mathrm{~W}$.

\section{Lithium carbonate and tetracycline} interaction

SIR,-Dr A J McGennis (6 May, p 1183) reports a case of lithium intoxication claimed to be a consequence of the nephrotoxic effect of tetracycline given concurrently. However, at least two other explanations of the intoxication reported seem just as reasonable.

It is a well-known fact that sodium plays a special role in the development and course of lithium intoxication. Recently Thomsen et al, ${ }^{1}$ on the basis of clinical experience and experiments with rats, postulated the following mechanism. Administration of even non-toxic doses of lithium leads to an inhibition of the renal response to mineralocorticoids such as aldosterone. This causes a lithium-induced inhibition of distal sodium reabsorption, a condition that increases the minimum sodium requirement and may remain stable. However change in sodium intake or in minimum sodium requirement from other causes may make this condition unstable. The minimum sodium requirement is determined by the serum lithium concentration. When this rises the sodium requirement increases. Sodium requirement is further increased as a result of extrarenal sodium loss like heavy sweating or diarrhoea. It is important to note that the "critical level" of serum lithium concentration that at which sodium requirement exceeds the total nicotine obtained, as estimated from Paper, Long Range Health Planning, Health and sodium intake, consequently does not have a fixed value. When the sodium intake is low (diet, intercurrent infections) it may fall below the minimum requirement and the critical serum lithium concentration is consequently low in these circumstances. This means that intoxication may occasionally develop at serum lithium concentrations within the therapeutic range in the absence of kidney disease. In the case reported sodium intake and loss are not mentioned. Therefore the possibility remains that the patient had a low critical serum lithium level owing to low sodium intake. The reported serum lithium levels of $0.5-0.86$ $\mathrm{mmol} / \mathrm{l}(0.35-0.5 \mathrm{mg} / 100 \mathrm{ml})$ may support such a hypothesis if these levels reflect changes in serum lithium concentration on a constant dose, thus indicating an unstable condition.

Another and probably more reasonable explanation is the concurrent use of tetracycline. In clinical practice diarrhoea is a more common side effect of orally given tetracycline than the nephrotoxic effect. It is therefore possible that the tetracycline caused diarrhoea with concomitant increased sodium loss and that this increased the sodium requirement above the critical level and lithium intoxication developed as a consequence. Also a combination of the two mechanisms described here may have been at work.

The present discussion emphasises that lithium therapy needs close supervision of the serum concentration even when the dose remains constant.

ULRIK MALT

Psychiatric Institute

\section{Thomsen, $\mathrm{K}$, et al, in Current Developments in Psychopharmacology, ed L Valzelli and W
Essman, vol 3, p 156. New York, Spectrum, 1976.}

\section{Hygiene of operating theatre cleaning equipment}

SIR,-We wish to draw attention to the importance of mops and buckets used for theatre cleaning as a possible source of nfection to patients. Not much has been written on this subject, but Thomas and Maurer' ${ }^{1}$ showed the importance of hygienic mop maintenance in operating theatres.

We investigated the problem in the operating theatres of a busy general hospital. In the theatres synthetic sponge mops were stored overnight in buckets containing a $1 \%$ phenolic solution. The same mop and bucket were used to clean the sluice and the theatre suite. Samples taken from bucket fluids were heavily contaminated by Pseudomonas aeruginosa. The heaviest contamination was found in the autoclave room bucket fluid, where an in-use test, using the Kelsey and Maurer ${ }^{2}$ method, showed $P s$ aeruginosa at a concentration of $>10^{6}$ organisms $/ \mathrm{ml}$.

Our domestic manager was at that time evaluating a new range of mops with autoclavable sponge heads and colour-matched buckets, which he suggested might effectively replace the old system. The idea was taken further by using colour-coded sets whose use was confined to specific areas within the theatre suites. ${ }^{3}$ Bacteriological monitoring of the new equipment showed a great improvement. No $P s$ aeruginosa was isolated from the mops and buckets provided both were stored dry and the mops upended. However, if the mops were stored in the $1 \%$ phenolic solution overnight or were stored downwards in the bucket there 